Acta Crystallographica Section D

Biological

Crystallography

ISSN 0907-4449

\section{Simon Parsons}

School of Chemistry, The University of Edinburgh, King's Buildings, West Mains Road, Edinburgh EH9 3JJ, Scotland

Correspondence e-mail: s.parsons@ed.ac.uk

(C) 2003 International Union of Crystallography

Printed in Denmark - all rights reserved

\title{
Introduction to twinning
}

A twinned crystal is an aggregate in which different domains are joined together according to a specific symmetry operation: the twin law. The diffraction patterns derived from different domains are rotated, reflected or inverted with respect to each other, depending on the nature of the relationship between the different domains, and weighted according to the quantity of a particular domain present in the crystal. The diffraction pattern measured during data collection is a superposition of all of these. Reflections from different domains may overlap and twinned crystals fall broadly into two categories in which either all reflections or only certain zones of reflections are affected by overlap. The former occurs when a crystal lattice belongs to a higher point group than the crystal structure itself; the latter frequently occurs when the twin law is a symmetry operation belonging to a higher symmetry supercell.

\section{Introduction}

Twinning is not an uncommon effect in crystallography, although it has long been considered to be one of the most serious potential obstacles to structure determination. The use of area detectors has much facilitated the detection of twinning and the treatment of diffraction patterns from twinned crystals. Computer software has also now been developed to such an extent that previously intractable twinning problems have yielded results of comparable precision to those obtained with untwinned samples. Structure determinations from twinned crystals are therefore becoming more common and the aim of this article is to present an introduction to the phenomenon of twinning. An extensive database of papers describing twinning has been assembled by Spek and Lutz (Utecht University, The Netherlands) and is available on the internet at http://www.cryst.chem.uu.nl/lutz/twin/ gen_twin.html. The review by Yeates (1997) will be of particular interest to macromolecular crystallographers.

\section{A simple model for twinning}

Twinning may occur when a unit cell (or a supercell) has higher symmetry than implied by the space group of the crystal structure. An example of a system which might be susceptible to twinning is a monoclinic crystal structure in $P 2_{1} / c$ where the unique angle, $\beta$, is equal or very close to $90^{\circ}$. In this case, the crystal structure has point group $2 / m$, but the lattice has point group $\mathrm{mmm}$. The elements of these point groups are

$$
2 / m: 1, m \perp \mathbf{b}, 2 \| \mathbf{b}, \overline{1},
$$

$m m m: 1, m \perp \mathbf{a}, 2\|\mathbf{a}, m \perp \mathbf{b}, 2\| \mathbf{b}, m \perp \mathbf{c}, 2 \| \mathbf{c}, \overline{1}$.
Received 23 January 2003 Accepted 7 August 2003 
The important issue is that $\mathrm{mmm}$ contains symmetry elements which do not occur in $2 / m$. Under these conditions, 'mistakes' can occur during crystal growth such that different regions of the crystal (domains) have their unit cells related by symmetry operations which are elements of $\mathrm{mmm}$ but not $2 / \mathrm{m}$ : a twofold axis about a, for example.

This idea can be illustrated by building up a stack of bricks. The overall shape or outline of a brick is $m m m$, but if we consider the 'dent' (brick-layers call this the frog) on one side plus the words 'London Brick', the point symmetry is only 2 (the 'space group' of this stack of bricks would be P2) (Fig. 1a). The most obvious way to build a stack of bricks is to place all the bricks in the same orientation, such as in Fig. 1(b): notice that the bricks are related to each other by the twofold axes perpendicular to the page or simple translation: both are elements of the space group. However, it is also possible to stack the bricks in such a way that some of the bricks are placed upside down (Fig. 1c). The overall shape of the brick, with the $90^{\circ}$ angles between the edges, allows this to happen without compromising the stacking of the bricks in any way. In turning some of the bricks upside down, we have used a twofold axis which is a symmetry operation of point group $m m m$ but not point group 2 .

Fig. $1(b)$ is similar to a single crystal; Fig. $1(c)$ resembles a twinned crystal. In Fig. 1(c) there are two domain variants: bricks (which correspond to unit cells) within the same domain are related to each other by translation; bricks in different domains are related by a translation plus a rotation which occurs in the point symmetry of the outline or overall shape of the brick. This extra symmetry operation corresponds in crystallography to the twin law. Had the extra element been chosen to be a mirror plane the mirror image of the words 'London Brick' would have appeared in the second domain and it is important to bear this in mind during the analysis of enantiopure crystals of chiral compounds (such as proteins). In protein crystallography the only possible twin laws are rotation axes. The fraction of the bricks in the alternative orientation corresponds to the twin scale factor, which in this example is 0.5 .

\section{Twinning in crystals}

Monoclinic crystal structures sometimes have $\beta$ very close to $90^{\circ}$. If twinning occurs, the unit cells in one domain may be rotated by $180^{\circ}$ about the $a$ or $c$ axes relative to those in the other domain in exactly the fashion described above for bricks. However, not all monoclinic crystal structures with $\beta \simeq$ $90^{\circ}$ form twinned crystals: twinning will only be observed if intermolecular interactions across a twin boundary are energetically competitive with those that would have been formed in a single crystal. For this reason, twinning very commonly occurs if a high-symmetry

Figure 1 phase of a material undergoes a transition to a lowersymmetry form upon change of temperature or pressure: a 'lost' symmetry element which made certain interactions equivalent in the high-symmetry form can act as a twin law in the low-symmetry form. Layered structures, such as the one shown in Fig. 2 (see also §7), are also often susceptible to twinning if the interactions between layers are rather weak and non-specific; alternative orientations of successive layers are then energetically similar. The total energy difference between intermolecular interactions which occur in a single, as opposed to a twinned, form of a crystal is one factor which controls the value of the domain scale factor, although in practice this may also be controlled kinetically by, for example, the rate of crystal growth.

In the foregoing discussion the impression might have been given that a twinned crystal consists of just two domains. A monoclinic crystal with $\beta \simeq 90^{\circ}$ twinned via a twofold rotation about a, though it contains two domain variants, may actually consist of very many domains. The orientations of the unit cells in any pair of domains will be related either by the identity operator or by the twin law. Further examples have been illustrated by Giacovazzo (1992) and other lead references can be found in the article by Koch (1992); an illustrative study of the domain structure of $\mathrm{KLiSO}_{4}$ has been given by Klapper et al. (1987).

Although the properties of a material (e.g. mechanical and optical properties) can depend strongly on domain structure, it is usually not necessary to characterize this for the purposes of ordinary structure analysis. However, the twin scale factor may appear to vary when different regions of a crystal are sampled during data collection. This can give rise to powerful non-isomorphism effects, as discussed by Terwisscha van Scheltinga et al. (2003).

\section{Diffraction patterns from twinned crystals}

Each domain of a twinned crystal gives rise to its own diffraction pattern; what is measured on a diffractometer is a

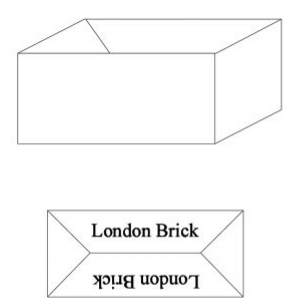

(a)

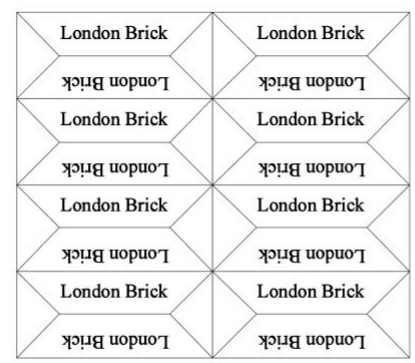

(b)

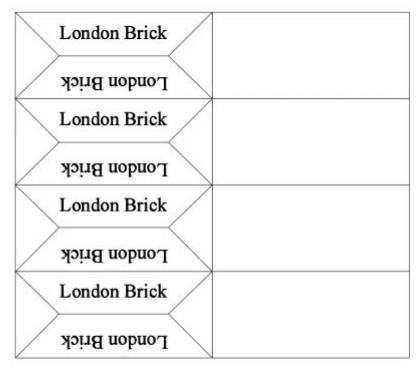

(c)
A simple model for twinning. (a) A brick; the top face of the brick has an indentation and the words 'London Brick' embossed on two sides of the indentation. (b) A stack of bricks where all the bricks are related to one another by translation. This resembles the relationship between units cells making up a single crystal. (c) Here some of the bricks have been placed upside down. The bricks still fit together because in turning a brick upside down we have used a symmetry element of the outline or overall shape of the brick. This resembles the relationship between unit cells in a twinned crystal. In both $(b)$ and $(c)$ the figures are intended to represent a whole crystal. 

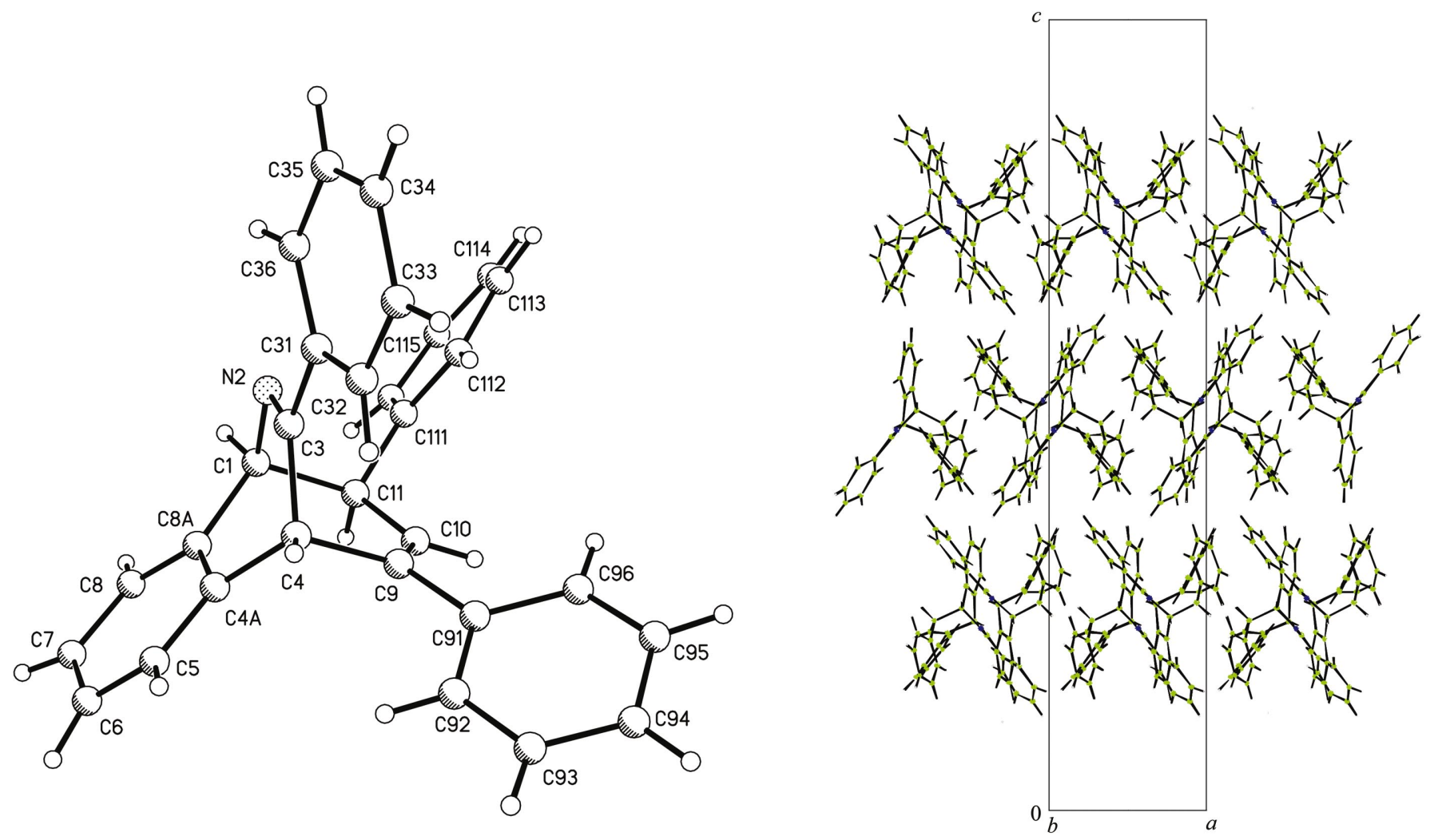

Figure 2

Molecular structure of compound (1). This is a monoclinic structure in which $\beta=90^{\circ}$, twinned via a twofold rotation about a.

superposition of all these patterns with intensities weighted according to the domain scale factors. The relative orientations of the diffraction patterns from different domains are the same as the relative orientations of the domains, so that if they are related by a $180^{\circ}$ rotation about a, then so too are their diffraction patterns. Fig. 3 shows this for a twinned monoclinic crystal structure for which $\beta=90^{\circ}$. Twinning is a problem in crystallography because it causes superposition or overlap between reflections which are not related by symmetry. In Fig. 3(c), the reflection which would have been measured with indices 102 is actually a superposition of the 102 reflection from domain 1 (Fig. $3 a$ ) and the $\overline{1} 02$ reflection from domain 2 (Fig. $3 b$ ). During structure analysis of a twinned crystal, it is important to define exactly which reflections contribute to a given intensity measurement: this is the role of the twin law.

In order to treat twinning during refinement, the twin law must obviously form part of the model. Usually, it is input into a refinement program in the form of a $3 \times 3$ matrix. In the example shown in Fig. 3 , the twofold axis about the $a$ axis will transform a into $\mathbf{a}, \mathbf{b}$ into $-\mathbf{b}$ and $\mathbf{c}$ into $-\mathbf{c}$. This is the transformation between the cells in different domains of the crystal; written as a matrix this is

$$
\left(\begin{array}{ccc}
1 & 0 & 0 \\
0 & -1 & 0 \\
0 & 0 & -1
\end{array}\right)
$$

The same matrix relates the indices of pairs of overlapping reflections, ${ }^{1}$

$$
\left(\begin{array}{ccc}
1 & 0 & 0 \\
0 & -1 & 0 \\
0 & 0 & -1
\end{array}\right)\left(\begin{array}{l}
h \\
k \\
l
\end{array}\right)=\left(\begin{array}{c}
h \\
-k \\
-l
\end{array}\right) .
$$

This two-component twin can be modelled using a quantity $\left|F_{\text {twin,calc }}\right|^{2}$, which is a linear combination (equation 1 , Pratt et al., 1971) consisting of $|F|^{2}$ terms for each component reflection weighted according to the twin scale factor, $x$, which can be refined,

$$
\begin{aligned}
\left|F_{\text {twin,calc }}(h, k, l)\right|^{2}= & (1-x)\left|F_{\text {calc }}(h, k, l)\right|^{2} \\
& +x\left|F_{\text {calc }}(h,-k,-l)\right|^{2}
\end{aligned}
$$

In the single-crystal reciprocal-lattice plots shown in Figs. 3(a) and $3(b)$, although twofold axes and mirror planes about the $a^{*}$ and $c^{*}$ axes relate the positions of the spots, this symmetry is not expressed in the intensities of the spots (for example, the $\overline{1} 02$ and 102 reflections have different intensities in Fig. $3 a$ ). However, in the composite twinned pattern (Fig. $3 c$ ), both the positions and the intensities of the spots exhibit the same mirror or twofold symmetry with respect to both of these axes. The composite pattern with equal domain volumes (that is $x=0.5$; Fig. $3 c$ ) appears to have orthorhombic diffraction

\footnotetext{
${ }^{\mathbf{1}}$ Here, the triple $h k l$ is represented as a column vector; if it is treated as a row vector (as it is in some software packages), the twin matrices discussed in this paper should be transposed.
} 
symmetry even though the crystal structure is monoclinic. In general, for a two-component twin, if $x$ is near 0.5 then merging statistics will appear to imply higher point symmetry than that possessed by the crystal structure. As $x$ deviates from 0.5 , then the merging in the higher-symmetry point group gradually becomes poorer relative to merging in the point group which corresponds to the space group of the crystal structure (Fig. $3 d$ ). Although it is impossible to give a definitive range, merging in the higher-symmetry point group may yield a merging residual of $0.10-0.35$; values of around 0.60 might be expected for untwinned samples (although pseudosymmetry in, for example, heavy-atom positions can give rise to a similar effect).

Another striking feature of the twinned diffraction pattern shown in Fig. 3(c) is that it appears to have a more acentric intensity distribution than the component patterns. The superposition of the diffraction patterns arising from the different domains tends to average out intensities because

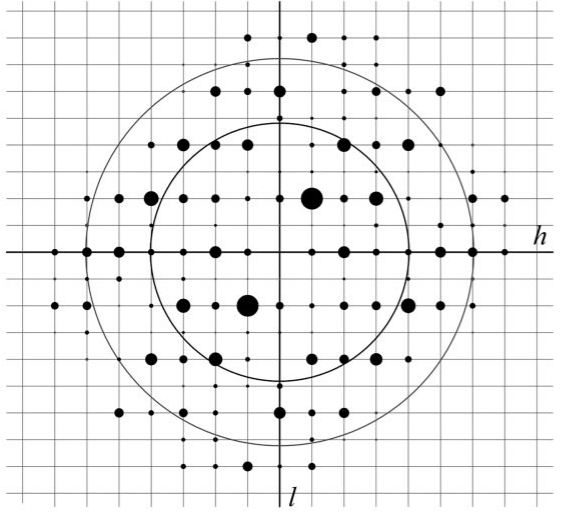

(a)

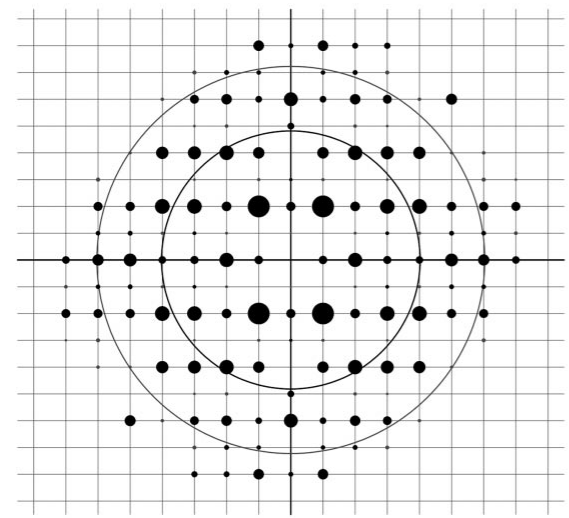

(c)

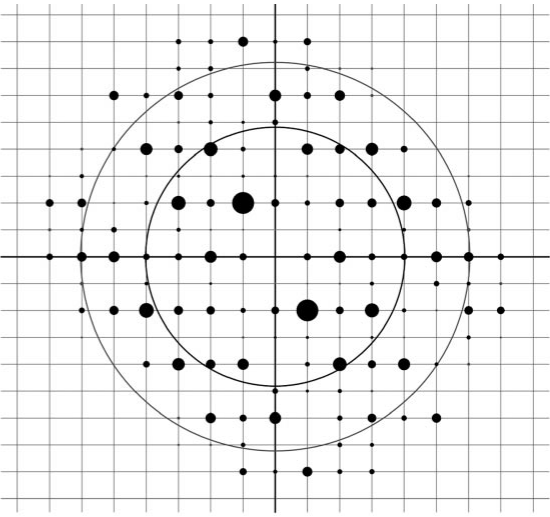

(b)

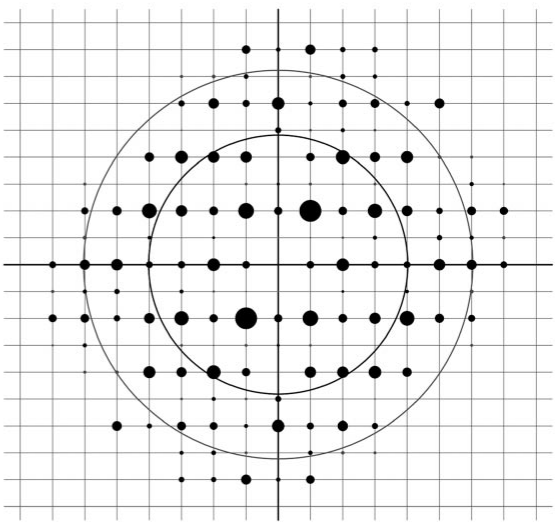

(d)
Figure 3

The effect of twinning by a twofold rotation about a on the diffraction pattern of a monoclinic crystal with $\beta=90^{\circ}$. Only the $h 0 l$ zone is illustrated; the space group is $P 2_{1} / c$. (a) $h 0 l$ zones from a singlecrystal. This could represent the diffraction pattern from one domain of a twinned crystal. $(b)$ This is the same pattern as shown in (a), but rotated about $\mathbf{a}^{*}$ (or $h$ axis) (which is coincident with the $a$ axis of the direct cell). This figure represents the diffraction pattern from the second domain of a twinned crystal. (c) Superposition of $(a)$ and $(b)$ simulating a twin with a domain scale factor of 0.5 ; that is, both domains are present in equal amounts. $(d)$ Superposition of $(a)$ and $(b)$ simulating a twin with a domain scale factor of 0.2 : the crystal consists on $80 \%$ of one domain $(a)$ and $20 \%$ of the other $(b)$. The values of $\left|E^{2}-1\right|$ for each figure are $(a)$ and $(b) 1.015,(c) 0.674,(d) 0.743$. The ideal (untwinned) value of $\left|E^{2}-1\right|$ for this centric crystal structure is 0.97 , meaning that its diffraction pattern characterized by the presence of both strong and weak reflections; intensities are more evenly distributed in acentric distributions, where $\left|E^{2}-1\right|$ has an ideal value of 0.74 . strong and weak reflections sometimes overlap. The quantity $\left|E^{2}-1\right|$, which adopts values of 0.97 and 0.74 for ideal centric twinning, although it is important to bear in mind all the usual caveats relating to the assumption of a random distribution of toms, which is not appropriate, for example, in the presence heavy atoms or non-crystallographic symmetry (Alexeev, The latter statistical tests will fail, however, for twins w near 0.5. If the value of $x$ is known and is not near 0.5 , (equation 1) can be used to 'detwin' a data set. This procedure may be useful for the purposes of structure solution, although it is generally preferable to refine against the original twinned data set.

Common signs of twinning have been listed by Herbst-Irmer \& Sheldrick (1998, 2002). Additional signs to those described above include an inability to solve a structure even though the data appear to be of good quality or, if a structure can be solved, a high $R$ factor or a noisy inexplicable difference electron-density map. Twinning also reveals itself in the Patterson function and this is discussed by Dauter (2003) and Yeates (1997). Since reflections from one domain may overlap with systematic absences from another, the observed systematic absences may either not be consistent with any known space group or appear to imply a very rare space group. Low-symmetry trigonal and hexagonal crystal structures appear to be particularly susceptible to twinning. It was pointed out by a referee to this paper that because twinning makes the point symmetry appear higher than it actually is, there seem to be more molecules in the unit cell than is actually the case, so that an unreasonably high packing density can also be taken to be a useful warning sign of twinning in macromolecular crystallography.

\section{Inversion, merohedral and pseudo-merohedral twins}

Twinning can occur whenever a compound crystallizes in a unit cell with a higher point group than that corresponding to the space group. This 
can occur for crystal structures in non-centrosymmetric space groups, since all lattices have inversion symmetry. Thus, a crystal of a compound in a space group such as $P 2_{1}$ may contain enantiomorphic domains (Flack, 2003). This type of twinning does not occur for an enantiopure compound and it can therefore be ruled out in protein crystallography. The twin law in this case is the inversion operator,

$$
\left(\begin{array}{ccc}
-1 & 0 & 0 \\
0 & -1 & 0 \\
0 & 0 & -1
\end{array}\right)
$$

This kind of twinning does not hamper structure solution and is most commonly encountered in Flack's method for absolute structure determination (Flack, 1983). The domain scale factor in this case is referred to as the Flack parameter.

Twinning may also occur in lower symmetry tetragonal, trigonal and cubic systems. Thus, a tetragonal structure in point group 4/m may twin about the twofold axis along [110], which is a symmetry element of the higher-symmetry tetragonal point group $4 / \mathrm{mmm}$. The twin law in this case is

$$
\left(\begin{array}{ccc}
0 & 1 & 0 \\
1 & 0 & 0 \\
0 & 0 & -1
\end{array}\right),
$$

this matrix may also be used in the treatment of low-symmetry trigonal, hexagonal and cubic crystal structures, producing diffraction patterns with apparent $\overline{3} m 1,6 / \mathrm{mmm}$ and $m \overline{3} m$ symmetry, respectively, when the domain scale factor $x$ is 0.5 .

Two further twin laws need to be considered in lowsymmetry trigonal crystals. A twofold rotation about [1 $\overline{1} 0]$, mimicking point group $\overline{3} 1 \mathrm{~m}$ when $x=0.5$, is expressed by the matrix

$$
\left(\begin{array}{ccc}
0 & -1 & 0 \\
-1 & 0 & 0 \\
0 & 0 & -1
\end{array}\right)
$$

By twinning via a twofold axis about [001], a trigonal crystal may also appear from merging statistics to be hexagonal if $x=0.5$. The twin law in this case is

$$
\left(\begin{array}{ccc}
-1 & 0 & 0 \\
0 & -1 & 0 \\
0 & 0 & 1
\end{array}\right) .
$$

In rhombohedral crystal structures, twinning of this type leads to obverse-reverse twinning.

The point groups of the crystal lattices ( $\overline{1}$ for triclinic, $2 / m$ for monoclinic, $\mathrm{mmm}$ for orthorhombic, $4 / \mathrm{mmm}$ for tetragonal, $\overline{3} m$ for rhombohedral, 6/mmm for hexagonal and $m \overline{3} m$ for cubic) are referred to as the holohedral point groups. Those point groups which belong to the same crystal family, but which are subgroups of relevant holohedral point group, are referred to as merohedral point groups (this classification is discussed in detail by Hahn \& Klapper, 1996). Thus, $4 / m$ is a merohedral point group of $4 / \mathrm{mmm}$. With the exception of obverse-reverse twinning (see below), in all the cases described in the previous paragraphs in this section the twin law was a symmetry operation of the relevant holohedry (i.e. of the crystal lattice) which was not expressed in the point symmetry corresponding to the crystal structure. For this reason, this type of phenomenon is referred to as twinning by merohedry. Such twins are often described as merohedral and although this usage is occasionally criticized in the literature (Catti \& Ferraris, 1976), it appears to have stuck. ${ }^{2}$ Though it is quite rare in molecular crystals, twins containing more than two domain variants are sometimes observed (see \$6); more commonly only two are present, however, and such twins are also described as hemihedral twins.

Twinning by merohedry should be carefully distinguished from the example described in $\$ 4$ where a monoclinic crystal structure accidentally had a $\beta$ angle near $90^{\circ}$; for example, there is nothing 'accidental' about a low-symmetry tetragonal structure having a lattice with symmetry $4 / \mathrm{mmm}$ : all lowsymmetry tetragonal structures have this property. Put another way, the holohedry of the tetragonal lattice is $4 / \mathrm{mmm}$; the low-symmetry tetragonal structure might belong to point group $4 / m, 4$ or $\overline{4}$, which are all nevertheless still tetragonal point groups; this is what would make this twinning by merohedry.

A trigonal crystal structure may be merohedrally twinned via a twofold axis about the [001] direction (parallel to the threefold axis), because this is a symmetry element of the 6/mmm holohedry. However, the rhombohedral lattice holohedry is $\overline{3} m$ and this point group does not contain a twofold axis parallel to the threefold axis. Although twinning via a twofold axis in this direction can certainly occur for rhombohedral crystal structures, it is not twinning by merohedry. Instead, it is referred to as obverse-reverse twinning or twinning by reticular merohedry; this is an important distinction because overlap between reflections from different domain variants in obverse-reverse twins only affects a third of the intensity data. This has recently been discussed in detail by Herbst-Irmer \& Sheldrick (2002).

A monoclinic crystal structure which happens to have $\beta \simeq 90^{\circ}$ has a lattice with, at least approximately, the $\mathrm{mmm}$ symmetry characteristic of the orthorhombic crystal family. If twinning occurs by a twofold axis about a or $\mathbf{c}$, the crystal is not merohedrally twinned, since monoclinic and orthorhombic are two different crystal families. This type of effect is instead referred to as twinning by pseudo-merohedry. A further example might occur in an orthorhombic crystal where two sides ( $b$ and $c$, say) are of equal length (pseudo-tetragonal). The twin law in this case could be a fourfold axis about a,

$$
\left(\begin{array}{ccc}
1 & 0 & 0 \\
0 & 0 & 1 \\
0 & -1 & 0
\end{array}\right) .
$$

A monoclinic crystal where $a \simeq c$ and $\beta \simeq 120^{\circ}$ may be twinned by a threefold axes about b. The clockwise and

\footnotetext{
${ }^{2}$ Holo and mero are Greek stems meaning whole and part, respectively. This 'French School' nomenclature was originally devised to describe crystal morphology and is used here because it is currently popular in the literature. Different nomenclature is also encountered; see, for example, Giacovazzo (1992) or van der Sluis (1989).
} 
anticlockwise threefold rotations $\left(3^{+}\right.$and $\left.3^{-}\right)$about this direction are

$$
\left(\begin{array}{ccc}
0 & 0 & 1 \\
0 & 1 & 0 \\
-1 & 0 & -1
\end{array}\right) \text { and }\left(\begin{array}{ccc}
-1 & 0 & -1 \\
0 & 1 & 0 \\
1 & 0 & 0
\end{array}\right)
$$

potentially yielding a three-component pseudo-merohedral twin appearing from the diffraction symmetry to be hexagonal.

Note that higher symmetry may be 'hidden' in a centred setting of a unit cell and not be immediately obvious from the cell dimensions and it is necessary to inspect carefully the output from whichever program has been used to check the metric symmetry of the unit cell [Herbst-Irmer \& Sheldrick (1998) have described two illustrations of this].

\section{Derivation of twin laws}

In $\S 4$ the case of a monoclinic crystal where $\beta \simeq 90^{\circ}$ was examined and it was shown that twinning could occur about a twofold axis about a. This leads to overlap between reflections with indices $h k l$ and $h \overline{k l}$. Twinning via a twofold axis about $\mathbf{c}$ would lead to overlap between reflections with indices $h k l$ and $\overline{h k} l$. However, since reflections $h \overline{k l}$ and $\overline{h k} l$ are related by the monoclinic twofold axis about $\mathbf{b}^{*}$ which must be present if the crystal point group is 2 or $2 / m$, these twin laws are equivalent. However, in the twinning about two threefold axes described in $\S 5$ for a monoclinic crystal with $a \simeq c$ and $\beta \simeq 120^{\circ}$, the rotations are not equivalent because they are not related by any of the symmetry operations of point group $2 / \mathrm{m}$.

It is usually the case that several equivalent descriptions may be used to describe a particular twin. However, several distinct twin laws may be possible and they can be expressed simultaneously. There clearly exists a potential for possible twin laws to be overlooked during structure analysis. Flack (1987) has described the application of coset decomposition to this problem, enabling this danger to be systematically avoided. The procedure has been incorporated by Litvin into the computer program TWINLAWS (Schlessman \& Litvin, 1995). ${ }^{3}$

Suppose that a crystal structure in point group $G$ crystallizes in a lattice with a higher point-group symmetry $H$. The number of possible twin laws is given by $\left(h_{H} / h_{G}\right)-1$, where $h_{G}$ and $h_{H}$ are the respective orders of point groups $G$ and $H$ (that is, the number of symmetry operations they contain). For example, in a protein crystallizing in point group 2 (space group $P 2, C 2$ or $P 2_{1}$ ) with a unit cell with parameters $a=30.5, b=30.5$, $c=44.9 \AA, \beta=90.02^{\circ}, G$ is point group 2 and $H$ is effectively point group $422(4 / \mathrm{mmm}$ in principle, but mirror symmetry is not permitted for an enantiopure protein crystal). The orders of $G$ and $H$ are 2 and 8, respectively, and so this crystal may suffer from up to three twin laws to form, at most, a twin with four domain variants (the reference domain plus three others).

\footnotetext{
${ }^{3}$ This program is available free of charge to academic users from http:// www.bk.psu.edu/faculty/litvin/Download.html or via the CCP14 web site (http://www.ccp14.ac.uk).
}

Table 1

Coset decomposition of point group 422 with respect to point group 2.

Output taken from the program TWINLAWS (Schlessman \& Litvin, 1995). The four rows represent the four different domains; either symmetry operation in a row may be taken to generate that domain.

\begin{tabular}{ll}
\hline 1 & $2(Y)$ \\
$2(X)$ & $2(Z)$ \\
$2(X-Y) \dagger$ & $4(Z)$ \\
$4(3)(Z) \ddagger$ & $2(X Y) \S$ \\
\hline
\end{tabular}

$\dagger$ The notation indicates a twofold rotation about the [110] direction. $\ddagger$ This is a $4^{-}$or $4^{3}$ rotation about [001]. \& This is a twofold rotation about [110].

Coset decomposition yields the symmetry elements which must be added to point group $G$ to form the higher point group $H$. Table 1 shows the output of the program TWIN$L A W S$, listing decomposition of point group 422 into cosets with point group 2. Possible twin laws are twofold axes about the [100], [110] and [110] directions. However, the twofold rotation about [110] is an equivalent twin law to the $4^{-}$(i.e. the $4^{3}$ ) rotation about [001] and the twofold axis about [100] is equivalent to that about [001].

The coset decomposition approach should clarify the rather complicated system of twin laws, presented in $\$ 5$, which apply to trigonal crystals. A trigonal crystal structure in $P 3, P 3_{1}$ or $P 3_{2}$ belongs to point group 3 (order 3), but is built on a lattice with $6 / \mathrm{mmm}$ symmetry (order 24). Twins with eight domain variants are possible in this case! The twin laws are formed by twofold rotations about [110], [11̄0] and [001], the inversion operator and mirror planes perpendicular to [110], [11̄0] and [001]. Of course, the last four of these can be ruled out in crystals of enantiopure chiral compounds and it should be stressed that such complicated multiple twinning is rather uncommon in molecular crystals.

\section{Examples of twinned crystal structures}

Crystals of the compound $\mathrm{C}_{30} \mathrm{H}_{27} \mathrm{~N}$ (1) (Fig. 2) diffracted rather weakly. The unit cell appeared to be orthorhombic with parameters $a=8.28, b=12.92, c=41.67 \AA$. The volume here fits for $Z=8$ and the value of $\left|E^{2}-1\right|$ was 0.725 . None of this is especially unusual, although the space group assuming orthorhombic symmetry appeared to be $P 22{ }_{1} 2$, which is rare. Merging statistics $\left(R_{\text {int }}\right)$, were as follows: $\mathrm{mmm}, 0.14 ; 2 / m, a$ unique, $0.13 ; 2 / m, b$ unique, $0.06 ; 2 / m, c$ unique, 0.09 . The lowest $R_{\text {int }}$ indicated monoclinic symmetry with the $b$ axis of the orthorhombic cell corresponding to the unique axis of the monoclinic cell. Taken with the space-group information described above this seemed to be a twin. The twin law used was

$$
\left(\begin{array}{ccc}
1 & 0 & 0 \\
0 & -1 & 0 \\
0 & 0 & -1
\end{array}\right)
$$

and space group $P 2_{1}$ was assumed. The structure did not solve by direct methods, but a position and orientation for one molecule (there are four in the asymmetric unit) was obtained by Patterson search methods (DIRDIF; Beurskens et al., 1996) 
using the rigid part of the molecule as a search fragment. The structure was completed by iterative cycles of least squares and Fourier syntheses (SHELXL97; Sheldrick, 1997). A search for missed space-group symmetry did not reveal any glide or mirror planes: the final $R$ factor was $10 \%$.

Further examples, more relevant to macromolecular structure determination, are given by Dauter (2003) and Terwisscha van Scheltinga et al. (2003). Worked examples for several twinning problems have been assembled by Herbst-Irmer and are available from http://shelx.uni-ac.gwdg.de/ rherbst/ twin.html.

\section{Non-merohedral twinning}

In merohedral and pseudo-merohedral twinning, the nature of the twin-law matrix means that all integral Miller indices are converted into other integer triples, so that all reciprocallattice points overlap. This usually means that all reflections are affected by overlap, although reflections from one domain may overlap with systematic absences from another. Twins in which only certain zones of reciprocal-lattice points overlap are classified as being non-merohedral. In these cases, only reflections which meet some special conditions on $h, k$ and/or $l$ are affected by twinning.

A non-merohedral twin law is commonly a symmetry operation belonging to a higher symmetry supercell. A simple example which might be susceptible to this form of twinning is an orthorhombic crystal structure where $2 a \simeq b$ (Fig. 4). A metrically tetragonal supercell can be formed by doubling the length of $a$ so that there is a pseudo-fourfold axis about c. The diffraction pattern from one domain of the crystal is related to that from the other by a $90^{\circ}$ rotation about $\mathbf{c}^{*}$. Superposition of the two diffraction patterns shows that data from the first domain are only affected by overlap with data from the second domain when $k$ is even (Fig. $4 d$ ). For the purposes of structure analysis, this twin law needs to be expressed with respect to the axes of the true orthorhombic cell. From Fig. 4(a), $\mathbf{a}^{\prime}=-0.5 \mathbf{b}, \mathbf{b}^{\prime}=2 \mathbf{a}, \mathbf{c}^{\prime}=\mathbf{c}$, so that the twin law is

$$
\left(\begin{array}{ccc}
0 & -0.5 & 0 \\
2 & 0 & 0 \\
0 & 0 & 1
\end{array}\right)
$$

The effect of this matrix on the data is

$$
\left(\begin{array}{ccc}
0 & -0.5 & 0 \\
2 & 0 & 0 \\
0 & 0 & 1
\end{array}\right)\left(\begin{array}{l}
h \\
k \\
l
\end{array}\right)=\left(\begin{array}{c}
-k / 2 \\
2 h \\
l
\end{array}\right),
$$

confirming that only data with $k=2 n$ are affected by the twinning. Thus, the 143 reflection from the first domain (green) is overlapped with the -223 reflection from the second (blue) domain. The 413 reflection in the green domain would be unaffected by twinning.

It is likely that the example given here would index readily on the tetragonal supercell, but notice the bizarre systematic absences in Fig. 4(d). Zones of unusual systematic absences are frequently a sign that a crystal is non-merohedrally twinned. This pseudotranslational symmetry should enable

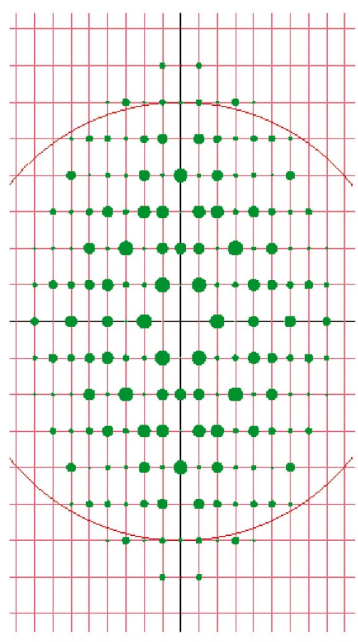

(b)

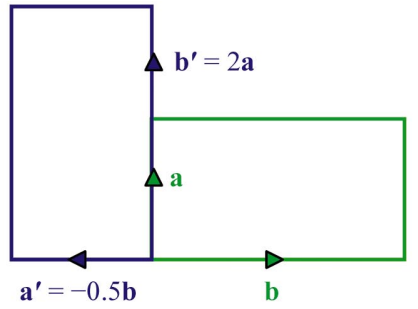

(a)

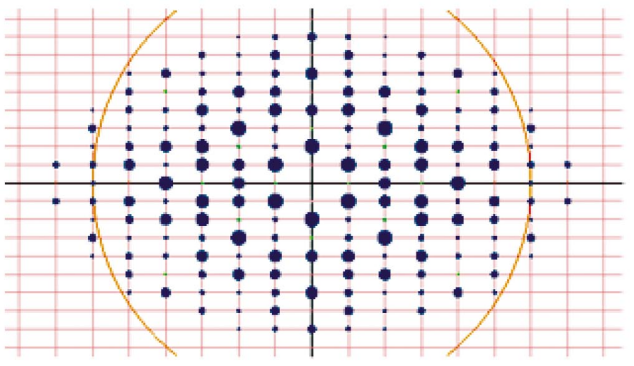

(c)

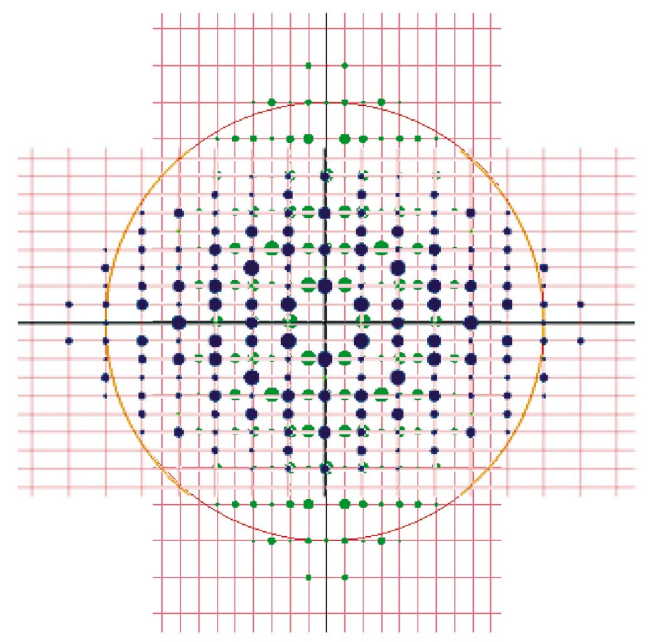

(d)

Figure 4

Non-merohedral twinning in an orthorhombic crystal where $2 a=b$. (a) The relationship of the unit cells in different domains is a $90^{\circ}$ rotation about $c$. $(b)$, $(c)$ Diffraction patterns from the two different domains in the crystal. The green spots in $(b)$ arise from cells in the orientation shown in green in $(a)$; likewise, the blue spots in $(c)$ come from the blue orientation in $(a)$. (d) Superposition of $(b)$ and $(c)$ to illustrate the diffraction pattern that would be measured for the twinned crystal. Note that blue and green spots only overlap where $k$ is an even number. Both Figs. 3 and 4 were drawn using $X P R E P$ (Sheldrick, 2001). 
the true orthorhombic cell to be inferred and it can be characterized in a Patterson synthesis.

In orthorhombic and higher systems, potential non-merohedral twins laws can often be derived from inspection of the unit-cell parameters. In low-symmetry crystals the twin law is usually less obvious (general procedures are given below), but it is possible to make a few general observations which apply to monoclinic crystals. In these cases, the twin law is often found to be a twofold axis about the unit cell $a$ or $c$ axes. The matrix for a twofold rotation about the $a$ axis is

$$
\left[\begin{array}{ccc}
1 & 0 & 0 \\
0 & -1 & 0 \\
(2 c \cos \beta) / a & 0 & -1
\end{array}\right] .
$$

The corresponding rotation about $c$ is

$$
\left[\begin{array}{ccc}
-1 & 0 & (2 a \cos \beta) / c \\
0 & -1 & 0 \\
0 & 0 & 1
\end{array}\right] .
$$

Likely twin laws can be derived for monoclinic crystals by evaluating the off-diagonal terms in these matrices; if nearrational values are obtained, the corresponding matrix should be investigated as a possible twin law.

\section{The derivation of non-merohedral twin laws}

Diffraction patterns from non-merohedrally twinned crystals contain many more spots than would be observed for an untwinned sample. Since individual spots may come from different domains of the twin, such diffraction patterns are frequently difficult to index. Overlap between reflections may be imperfect in some or all zones of data affected and integration and data reduction needs to be performed carefully. Software for integrating data sets from non-merohedral twins and performing absorption corrections has recently become available [for example, SAINT (Bruker-Nonius, 2002); EVAL-14 (Duisenberg et al., 2003) and TWINABS (Sheldrick, 2002)].

Excellent programs such as DIRAX (Duisenberg, 1992) and GEMINI (Sparks, 1999) have been developed to index diffraction patterns from non-merohedral twins. In many cases a pattern can be completely indexed with two orientation matrices and both these programs offer procedures by which the relationship between these alternative matrices is analysed to suggest a twin law. It is usually the case that twinning can be described by a twofold rotation about a direct or reciprocallattice direction. Indeed, if two such directions are parallel and the vectors describing them have a dot product of greater than two, then a higher-symmetry supercell can be derived. The program CREDUC (Le Page, 1982) is extremely useful for investigating this; it is available in the Xtal suite of software (Hall et al., 1992), which can be downloaded from http:// www.ccp14.ac.uk; the algorithm is also used in the LEPAGE routine in PLATON (Spek, 2003). A related program, called $O B L I Q U E$, has recently been described by Le Page (2002).

It is sometimes the case that the first intimation that the analyst has that a crystal is twinned is during refinement.
Symptoms such as large inexplicable difference Fourier map peaks and a high $R$ factor may indicate that twinning is a problem, while careful analysis of poorly fitting data reveals that they belong predominantly to certain distinct zones in which $\left|F_{\text {obs }}\right|^{2}$ is systematically larger than $\left|F_{\text {calc }}\right|^{2}$. If twinning is not taken into account, it is likely that these zones are being poorly modelled and that trends in their indices may provide a clue to a possible twin law. The computer program ROTAX (Cooper et al., 2002; also available from http:// www.ccp14.ac.uk) makes use of this idea to identify possible twins laws. A set of data with the largest values of $\left[\left|F_{\text {obs }}\right|^{2}-\left|F_{\text {calc }}\right|^{2}\right] / u\left(\left|F_{\text {obs }}\right|^{2}\right.$ ) (where $u$ is the standard uncertainty of $\left.\left|F_{\text {obs }}\right|^{2}\right)$ is identified and the indices transformed by twofold rotations or other symmetry operations about possible direct and reciprocal-lattice directions. Matrices which transform the indices of the poorly fitting data to integers are identified as possible twin laws. The analyst then has a set of potential matrices which might explain the source of the refinement problems described above. A related procedure is available in the TwinRotMat routine in PLATON, but this uses a set of reflections from planes with similar $d$ spacings rather than high values of $\left[\left|F_{\text {obs }}\right|^{2}-\left|F_{\text {calc }}\right|^{2}\right] / u\left(\left|F_{\text {obs }}\right|^{2}\right)$.

\section{Non-merohedral twinning: an example<smiles>Oc1cccn(-c2ccccc2)c1=S</smiles>

(2)
Crystals of (2) grew as coaxially aligned aggregates of needles (Smith, 2000). The diffraction pattern was completely indexed using DIRAX using two orientation matrices. Some reflections in the search list gave integral indices with only one of these matrices, while others could be satisfactorily indexed with both. The crystal system was monoclinic $P, a=7.28, b=9.74$, $c=15.23 \AA$, $\beta=94.39^{\circ}$, space group $P 2_{1} / n$. We describe below how the program ROTAX can be used to evaluate the twin law; however, the same matrix could also be obtained with the program $2 V I E W$, which is part of the DIRAX system. Data were collected using one of the matrices derived by $D I R A X$. The structure was solved by Patterson methods (DIRDIF) and refinement (CRYSTALS; Watkin et al., 2002) converged to $R=14.8 \%$, with anisotropic displacement parameters and all $\mathrm{H}$ atoms except that attached to the hydroxyl group placed in calculated positions. The difference synthesis maximum of 1 e $\AA^{-3}$ was close to the $S$ atom, but in a chemically implausible position.

The program ROTAX has been incorporated into the CRYSTALS refinement suite, the application and refinement of a twinning model being facilitated by a GUI. All poorly fitting data had $h=3 n$ and the twin law was readily identified as the matrix 


$$
\left(\begin{array}{ccc}
-1 & 0 & 0 \\
0 & -1 & 0 \\
0.33 & 0 & 1
\end{array}\right)
$$

which corresponds to a twofold axis about the [106] direct lattice direction. This acts to overlap reflections $h k l$ with $h=3 n$ from one domain with reflections $-h,-k,(h / 3+l)$ from the other domain. Incorporation of this twin law into the refinement cleaned up the difference map considerably, allowing the hydroxyl $\mathrm{H}$ atom to be located. The final $R$ factor was $5.16 \%$, with a twin scale factor of 0.437 (3).

In the case of this crystal, a supercell can be obtained by application of the matrix

$$
\left(\begin{array}{ccc}
1 & 0 & 0 \\
0 & -1 & 0 \\
1 & 0 & 6
\end{array}\right)
$$

which transforms the true monoclinic cell to a metrically orthorhombic cell with dimensions $a=7.29, b=9.74$, $c=91.12 \AA$. It is important to attempt to identify the metric symmetry of the supercell because the fact that this cell is orthorhombic and no higher implies that there is no need to search for further twin laws. Decomposition of $\mathrm{mmm}$ into cosets with subgroup $2 / m$ yields the identity operator and either a twofold axis about $\mathbf{a}$ or $\mathbf{c}$ of the supercell, which has, of course, already been identified.

Further examples of non-merohedral twinning problems are given by Dauter (2003), Choe et al. (2000), Colombo et al. (2000), Gaudin et al. (2000), Guelylah et al. (2001), Cooper et al. (2002) and Tang et al. (2001). A worked example (Herbst-Irmer \& Sheldrick, 1998) is available from http:// shelx.uni-ac.gwdg.de/ rherbst/twin.html.

Note added in proof: Padilla \& Yeates (2003) have very recently described a statistical test for the detection of hemihedral twinning.

It is a pleasure to thank Richard Cooper, Howard Flack, Bob Gould and David Watkin for a very fruitful collaboration on twinning; Howard Flack deserves particular thanks for reading and commenting on the manuscript of this paper. Funding by the EPSRC and The University of Edinburgh is also gratefully acknowledged.

\section{References}

Alexeev, D. (2003). CCP4 Study Weekend on Experimental Phasing, January 2003, York, England.

Beurskens, P. T., Beurskens, G., Bosman, W. P., de Gelder, R., GarciaGranda, S., Gould, R. O., Israel, R. \& Smits, J. M. M. (1996). The DIRDIF96 Program System. Technical Report of the Crystallography Laboratory, University of Nijmegen, The Netherlands.

Britton, D. (1972). Acta Cryst. A28, 296-297.

Bruker-Nonius (2002). SAINT, version 7. Bruker-Nonius, Madison, Wisconsin, USA.

Catti, M. \& Ferraris, G. (1976). Acta Cryst. A32, 163-165.
Choe, W., Pecharsky, V. K., Pecharsky, A. O., Gschneidner, K. A., Young, V. G. \& Miller, G. J. (2000). Phys. Rev. Lett. 84, 46174620.

Colombo, D. G., Young, V. G. \& Gladfelter, W. L. (2000). Inorg. Chem. 39, 4621-4624.

Cooper, R. I., Gould, R. O., Parsons, S. \& Watkin, D. J. (2002). J. Appl. Cryst. 35, 168-174.

Dauter, Z. (2003). Acta Cryst. D59, 2004-2016.

Duisenberg, A. J. M. (1992). J. Appl. Cryst. 25, 92-96.

Duisenberg, A. J. M., Kroon-Batenburg, L. M. J. \& Schreurs, A. M. M. (2003). J. Appl. Cryst. 36 220-229.

Flack, H. D. (1983). Acta Cryst. A39, 876-881.

Flack, H. D. (1987). Acta Cryst. A43, 564-568.

Flack, H. D. (2003). Helv. Chim. Acta, 86, 905-921.

Gaudin, E., Petricek, V., Boucher, F., Taulelle, F. \& Evain, M. (2000). Acta Cryst. B56, 972-979.

Giacovazzo, C. (1992). Editor. Fundamentals of Crystallography. Oxford University Press.

Guelylah, A., Madariaga, G., Petricek, V., Breczewski, T., Aroyo, M. I. \& Bocanegra, E. H. (2001). Acta Cryst. B57, 221-230.

Hahn, T. \& Klapper, H. (1996). International Tables for Crystallography, Vol. A, edited by T. Hahn, ch. 10. Dordrecht: Kluwer Academic Publishers.

Hall, S. R., Flack, H. \& Stewart, R. F. (1992). Xtal3.2. Universities of Western Australia, Australia, Geneva, Switzerland and Maryland, USA.

Herbst-Irmer, R. \& Sheldrick, G. M. (1998). Acta Cryst. B54, 443-449.

Herbst-Irmer, R. \& Sheldrick, G. M. (2002). Acta Cryst. B58, 477-481. Kahlenberg, V. (1999). Acta Cryst. B55, 745-751.

Klapper, H., Hahn, T. \& Chung, S. J. (1987). Acta Cryst. B43, 147-159. Koch, E. (1992). International Tables for Crystallography, Vol. C, edited by A. J. C. Wilson, pp. 10-14. Dordrecht: Kluwer Academic Publishers.

Le Page, Y. (1982). J. Appl. Cryst. 15, 255-259.

Le Page, Y. (2002). J. Appl. Cryst. 35, 175-181.

Pratt, C. S., Coyle, B. A. \& Ibers, J. A. (1971). J. Chem. Soc. 21462151.

Padilla, J. E. \& Yeates, T. O. (2003). Acta Cryst. D59, 1124-1130.

Rees, D. C. (1980). Acta Cryst. A36, 578-581.

Schlessman, J. \& Litvin, D. B. (1995). Acta Cryst. A51, 947-949.

Sheldrick, G. M. (1997). SHELXTL97. University of Göttingen, Germany.

Sheldrick, G. M. (2001). XPREP. Bruker-AXS, Madison, Wisconsin, USA.

Sheldrick, G. M. (2002). TWINABS. Bruker-AXS, Madison, Wisconsin, USA.

Smith, A. (2000). PhD thesis, The University of Edinburgh, Edinburgh, Scotland.

Sparks, R. A. (1999). GEMINI. Bruker AXS, Madison, Wisconsin, USA.

Spek, A. L. (2003). PLATON: A Multipurpose Crystallographic Tool. Utrecht University, Utrecht, The Netherlands.

Tang, C. Y., Coxall, R. A., Downs, A. J., Greene, T. M. \& Parsons, S. (2001). J. Chem. Soc. Dalton Trans., pp. 2141-2147.

Terwisscha Van Scheltinga, A. C., Valegård, K., Hadju, J. \& Andersson, I. (2003). Acta Cryst. D59, 2017-2022.

Van der Sluis, P. (1989). Thesis, University of Utrecht, Utrecht, The Netherlands.

Watkin, D. J., Prout, C. K., Carruthers, J. R., Betteridge, P. W. \& Cooper, R. I. (2002). CRYSTALS. Issue 11. Chemical Crystallography Laboratory, University of Oxford, England.

Yeates, T. O. (1988). Acta Cryst. A44, 142-144.

Yeates, T. O. (1997). Methods Enzymol. 276, 344-358. 